\title{
Effect of the Height of a Wheelchair on the Shoulder and Forearm Muscular Activation During Wheelchair Propulsion
}

\author{
SANG-YeOL LeE, PhD, $\mathrm{PT}^{1)}$, SEON-ChIL KIM, $\mathrm{PhD}^{2)}$, \\ Myoung-HeE LEE, PhD, PT ${ }^{3)}$, JAE-SEONG Yoo $^{4}$ \\ 1) Department of Physical Therapy, Gimhea College University: Sambang-dong, Gimhea, \\ Kyeongsangnam-do, Republic of Korea, San 77-9. TEL: +82 55-320-1735, FAX: +82 54-442-8953, \\ E-mail:sjslh486@hanmail.net \\ 2) Department Radiologic Technology, Daegu Health College \\ 3) Department of Physical Therapy, Daegu Health College \\ 4) Korea Health Industry Development Institute, Support Center for Senior-Friendly Industry
}

\begin{abstract}
Purpose] The activations of the shoulder muscle and forearm muscle were analyzed at different chair heights with reference to the user's elbow joint flexion angle to provide fundamental kinematic data for the fabrication of wheelchairs suitable for the elderly and the disabled with spinal damage. [Subjects] Participants who met the criteria for this study ( $\mathrm{n}=12)$. [Methods] Muscular activity was measured during propulsion of a wheelchair with chair heights corresponding to elbow flexion angles of 0,30,60 and 90 degrees. To eliminate the effect of muscular fatigue, that might have been caused during wheelchair propulsion. [Results] The biceps brachii, anterior deltoid, pectoralis major, serratus anterior and latissimus dorsi muscle activation showed significant differences. [Conclusion] On the basis of the results of this study, we consider that excessive load on the shoulder muscles should be reduced by producing wheelchairs with adjustable chair heights and by using a wheelchair with consideration for an individual's body size. Future studies should be conducted on the somatic muscle activation at different on chair heights and on the appropriate chair height for various external environments such as a slopeway. Key words: Muscle activation, Wheelchair propulsion
\end{abstract}

(This article was submitted Jul. 21, 2011, and was accepted Aug. 30, 2011)

\section{INTRODUCTION}

In order to improve the quality of life of the disabled, their access to society should be guaranteed, and to this end, their mobility should be secured in advance ${ }^{1)}$.

The wheelchair is the most widely used aid to secure the mobility of the disabled. It is used not only to promote mobility, but also to prevent the user from being disabled and to compensate and correct disability. Since the wheelchairs are used mostly by patients with spinal damage, somatic stability has been studied. In particular, impaired and weakened ability to control the body during wheelchair propulsion reduces the somatic stability and causes kyphosis in disabled persons with spinal damage ${ }^{2)}$. An inappropriate position when using a wheelchair may cause back pain since it makes the user depend on the supportive surface when balance is lost in the wheelchair Back pain decreases the propulsive force of the body and the shoulder muscles have to be used to compensate. Hence, injuries to the shoulders, such as the rotator cuff injury, are caused $^{3,4)}$. In this way, the inappropriate use of a wheelchair causes various problems to the musculoskeletal system of the user.
Recently, wheelchairs are being used not only by the disabled with spinal damage but also by the elderly who have difficulty in moving, to increase their mobility. The weakening of the somatic muscles and degeneration of the shoulder articulations through spinal degeneration causes back pain and shoulder pain making it difficult to use the trunk muscles, shoulder muscles and forearm muscles.

Since wheelchairs are being used not only by the disabled with spinal damage but also by the elderly, wheelchairs should be produced to provide a structure which is appropriate for the condition of the users. However, among the various wheelchair propulsion factors, sufficient study of the height between the chair and the wheels, that is directly related with the propulsive force. In this study has not been conducted, therefore, activation of the shoulder muscles and forearm muscle was analyzed at different chair heights which were determined with reference to user elbow joint flexion angle to provide fundamental kinematic data for the fabrication of wheelchairs suitable for the elderly and the disabled with spinal damage. 
Table 1. Comparison of muscle activation according to elbow flexion angle when using a

\begin{tabular}{ccccc}
\multicolumn{3}{c}{ wheelchair } & (unit : \%RVC) \\
\hline & $0^{\circ}$ & $30^{\circ}$ & $60^{\circ}$ & $90^{\circ}$ \\
\hline AD* $^{\circ}$ & $10481.4 \pm 1863.7^{\mathrm{a}}$ & $7469.4 \pm 1484.9^{\mathrm{a}}$ & $5714.5 \pm 685.5^{\mathrm{b}}$ & $5074.6 \pm 637.7^{\mathrm{b}}$ \\
$\mathrm{PM}^{*}$ & $5791.0 \pm 1057.0^{\mathrm{a}}$ & $4809.7 \pm 1238.7^{\mathrm{a}}$ & $3171.9 \pm 489.1^{\mathrm{b}}$ & $2630.9 \pm 217.6^{\mathrm{b}}$ \\
$\mathrm{SA}^{*}$ & $3064.7 \pm 521.5^{\mathrm{a}}$ & $2095.4 \pm 335.3^{\mathrm{a}}$ & $1556.4 \pm 283.0^{\mathrm{b}}$ & $1371.6 \pm 216.0^{\mathrm{b}}$ \\
$\mathrm{BB}^{*}$ & $8545.0 \pm 1424.3^{\mathrm{a}}$ & $4868.6 \pm 764.5^{\mathrm{b}}$ & $3992.3 \pm 644.5^{\mathrm{b}}$ & $3747.7 \pm 534.9^{\mathrm{b}}$ \\
$\mathrm{FCR}$ & $3952.0 \pm 467.1$ & $3926.6 \pm 613.6$ & $3824.6 \pm 583.1$ & $4412.5 \pm 909.3$ \\
LD* & $1952.7 \pm 500.0^{\mathrm{a}}$ & $1603.8 \pm 520.3^{\mathrm{a}}$ & $671.6 \pm 179.5^{\mathrm{b}}$ & $700.6 \pm 180.4^{\mathrm{b}}$ \\
$\mathrm{ECR}$ & $6369.0 \pm 754.5$ & $6671.4 \pm 871.2$ & $6052.2 \pm 473.6$ & $7136.1 \pm 860.8$ \\
Tri & $5636.2 \pm 974.7$ & $5829.3 \pm 1184.5$ & $5239.5 \pm 876.5$ & $6017.3 \pm 1239.0$ \\
\hline
\end{tabular}

mean \pm SE. AD : anterior deltoid, PM : pectoralis major, SA : serratus anterior, $\mathrm{BB}:$ biceps brachii, FCR : flexor carpi radialis, LD : latissimus dorsi, ECR : extensor carpi radialis, Tri : triceps.

NOTE. Each value represents the mean \pm SE. The values with different superscripts $(a, b)$ in the same column are significantly different $(\mathrm{p}<0.05)$ by Tukey's measure. ${ }^{*}$ : statistically significant, $\mathrm{p}<0.05$.

\section{SUBJECTS AND METHODS}

The subjects of this study were 20 normal adults in their twenties who had no musculoskeletal disease and who had normal ROM. A sufficient explanation of the experimental procedures was provided to the subjects and the written consents indicating voluntary participation were received from them.

To limit the difficulty of wheelchair propulsion in each condition measurements were performed while propelling the wheelchair over a distance of $10 \mathrm{~m}$ at maximum speed and the mean measurement values were used in the analysis. To evaluate the muscular activity while propelling the wheelchair at different chair heights, the chair height was determined with reference to the elbow joint flexion angle when the user sat on the chair and held the highest part of the wheelchair wheels. The muscular activity was measured while propelling the wheelchair using chair heights corresponding to elbow flexion angles of $0^{\circ}, 30^{\circ}$, $60^{\circ}$ and $90^{\circ}$. To eliminate the effect of muscular fatigue, that might have been caused during the wheelchair propulsion, chair heights corresponding to the four elbow joint angles were randomly used in the experiment.

To collect accurate electromyographic data, epilation was carried out with a razor on the sites to which the electrodes were attached, and horny substance was removed with sandpaper. Then, the sites were cleansed with an alcohol swab. The electrodes to the attached were pectoralis major, flex carpi radialis, extensor carpi radialis, serratus anterior, anterior deltoid, biceps brachii, triceps and lattisimus dorsi, because these muscles are associated with stability of the shoulder.

ProComp InfinitiTM (Thought Technology Ltd., Canada) was employed for the measurement of the muscle activation of each muscle. A surface electrode (Triode surface electrode, Thought Technology Ltd., Canada) consisting of a tripolar electrode (positive-ground-negative) was used. The frequency range of the electromyogram signals was set to 20 to $500 \mathrm{~Hz}$, and the sampling frequency was set to $1024 \mathrm{~Hz}$.

The root mean square of each muscle was measured for five seconds in the anatomical position. The relative muscle contraction was calculated as the mean electromyogram signal measured during the middle three seconds, excluding the data of the first one second and the last one second, and expressed as the muscle activation in $\%$ RVC for $10 \mathrm{~m}$ performance of wheelchair propulsion.

The measured data was analyzed by one-way ANOVA using SPSS (version 12.0) for Windows to compare the muscle activations at the different chair heights. The level of significance was chosen as 0.05 .

\section{RESULTS}

The anterior deltoid muscle activity was 10481.4 at $0^{\circ}$, 7469.4 at $30^{\circ}, 5714.5$ at $60^{\circ}$, and 5074.6 at $90^{\circ}$ with significant differences $(\mathrm{p}<0.05)$. Pectoralis major muscle activation was 5791.0 at $0^{\circ}, 4809.7$ at $30^{\circ}, 3171.9$ at $60^{\circ}$ and 2630.9 at $90^{\circ}$ with significant differences $(\mathrm{p}<0.05)$. Serratus anterior muscle activity was 3064.7 at $0^{\circ}, 2095.44$ at $3^{\circ}, 1556.45$ at $60^{\circ}$, and 1371.6 at $90^{\circ}$ with significant differences $(\mathrm{p}<0.05)$. Latissimus dorsi muscle activity was 1952.7 at $0^{\circ}, 1603.8$ at $30^{\circ}, 671.6$ at $60^{\circ}$, and 700.6 at $90^{\circ}$ with significant differences $(\mathrm{p}<0.05)$. The post-hoc test found statistically significant differences between $0^{\circ}$ and $60^{\circ}, 0^{\circ}$ and $90^{\circ}, 30^{\circ}$ and $60^{\circ}, 30^{\circ}$ and $90^{\circ}$ for the anterior deltoid, pectoralis major, serratus anterior and latissimus dorsi muscles $(\mathrm{p}<0.05)$.

Biceps brachii muscle activation was 8545.0 at $0^{\circ}$, 4868.6 at $30^{\circ}, 3992.3$ at $60^{\circ}, 3747.7$ at $90^{\circ}$ with significant differences $(p<0.05)$. The post-hoc test found statistically significant differences between $0^{\circ}$ and $30^{\circ}, 60^{\circ}$ and $90^{\circ}$ for the biceps brachii muscle $(\mathrm{p}<0.05)$.

\section{DISCUSSION}

There is increasing interest in the wheelchair as a device for mobility in order to enhance the access of the increasing number of the elderly and the disabled to society. A wheelchair is used not only as a mobility device but also to prevent, compensate or correct disability, and inappropriate use of a wheelchair may adversely affect the remaining physical abilities of the elderly and the disabled.

The purpose of this study was to provide fundamental 
data for the development of wheelchairs with chair heights that can be adjusted to individual body sizes, because current wheelchairs are fixed and produced without consideration for individual body sizes.

We analyzed upper limb muscular activity during the propulsion of wheelchairs on flat ground at different chair heights. The activities of the anterior deltoid, pectoralis major, serratus anterior and latissimus dorsi were drastically decreased as the elbow flexion angle increased over 30 degrees. This indicates that the propulsion force was provided more by the shoulder muscles than by the elbow muscles as the chair height increased. Hence, to prevent the excessive use of the shoulder muscles, the appropriate chair height is the height that results in an elbow flexion angle between $60^{\circ}$ and $90^{\circ}$.

We also found that the wrist muscle and triceps were activated at all the different chair heights during propulsion of the wheelchair. This indicates that a constant load is delivered to the wrist joint regardless of the chair height when propelling a wheelchair. Thus, reduction of the shoulder joint load through changing the chair height or another structure of the wheelchair is the only method for decreasing the muscle fatigue caused by wheelchair use.
On the basis of the results of this study, we consider that it is possible to reduce the load on the shoulder muscles by producing a wheelchair with an adjustable chair height and by using with consideration for an individual's body size. Future studies should be conducted on the somatic muscle activation depending on the chair height and of the appropriate chair heights for various external environments such as a ramp.

\section{REFERENCES}

1) Roders MM, McQuade KJ, Rasch EK, et al.: Upper-limb fatique-related joint power shifts in experienced wheelchair users and nonwheelchair users. J Rehabil Res Dev, 2003, 40: 27--38

2) Hobson DA, Tooms RE: Seated lumbar/pelvic alignment. A comparison between spinal cord-injured and noninjured group. Spine, 1992, 17: 293 298

3) Samuelsson KA, Tropp H, Nylander E, et al.: The effect of rear-wheel position on seating ergonmics and mobility efficiency in wheelchair users whith spinal cord injuries: A pilot study. J Rehabil Res Dev, 2004, 41: 6574.

4) Sinnott KA, Milburn P, McNaughton H: Factors associated with thoracic spinal cord injury, lesion level and rotator cuff disorders. Spinal Cord, 2000, 38: $748-753$ 